Mens

revue d'histoire intellectuelle de l'Amérique française

\title{
L'évolution des sciences au Canada français examinée à partir de la mycologie
}

\section{Guy Gaudreau}

Volume 11, numéro 2, printemps 2011

URI : https://id.erudit.org/iderudit/1023372ar

DOI : https://doi.org/10.7202/1023372ar

Aller au sommaire du numéro

\section{Éditeur(s)}

Centre de recherche en civilisation canadienne-française

ISSN

1492-8647 (imprimé)

1927-9299 (numérique)

Découvrir la revue

Citer cet article

Gaudreau, G. (2011). L'évolution des sciences au Canada français examinée à partir de la mycologie. Mens, 11(2), 37-67. https://doi.org/10.7202/1023372ar
Résumé de l'article

L'article traite de l'évolution de la mycologie pratiquée au Canada français depuis le milieu du XIX ${ }^{\mathrm{e}}$ siècle. Sous l'angle de cette discipline spécifique que représente la mycologie, c'est la pratique des sciences qui est examinée, tout comme le rôle central joué par le frère Marie-Victorin et par certaines communautés religieuses, tels les Frères des écoles chrétiennes. En participant à la création des Cercles des jeunes naturalistes, terreau des futurs membres des clubs de mycologie, et en faisant la promotion des recherches et des études américaines et nord-américaines, Marie-Victorin joue vraisemblablement un rôle déterminant dans la carrière de nombreux scientifiques dont René Pomerleau, qui se taillera une place de choix sur la scène mycologique. Comme on le verra, ce dernier n'a pas été le seul à contribuer à l'évolution de la mycologie, même s'il est généralement présenté comme le fondateur des deux premiers cercles de mycologues amateurs. Une attention particulière sera portée au Cercle des mycologues amateurs de Montréal, fondé en 1950, et dont on suivra à grands traits l'évolution pendant quelques décennies. 


\title{
L'évolution des sciences au Canada français examinée à partir de la mycologie ${ }^{1}$
}

\author{
Guy Gaudreau \\ Université Laurentienne
}

\section{Résumé}

L'article traite de l'évolution de la mycologie pratiquée au Canada français depuis le milieu du XIX ${ }^{e}$ siècle. Sous l'angle de cette discipline spécifique que représente la mycologie, c'est la pratique des sciences qui est examinée, tout comme le rôle central joué par le frère MarieVictorin et par certaines communautés religieuses, tels les Frères des écoles chrétiennes. En participant à la création des Cercles des jeunes naturalistes, terreau des futurs membres des clubs de mycologie, et en faisant la promotion des recherches et des études américaines et nord-américaines, Marie-Victorin joue vraisemblablement un rôle déterminant dans la carrière de nombreux scientifiques dont René Pomerleau, qui se taillera une place de choix sur la scène mycologique. Comme on le verra, ce dernier n'a pas été le seul à contribuer à l'évolution de la mycologie, même s'il est généralement présenté comme le fondateur des deux premiers cercles de mycologues amateurs. Une attention particulière sera portée au Cercle des mycologues amateurs de Montréal, fondé en 1950, et dont on suivra à grands traits l'évolution pendant quelques décennies.

\footnotetext{
${ }^{1}$ Nous tenons à remercier les quatre évaluateurs anonymes de la revue pour leurs précieuses remarques, de même que notre collègue botaniste Jacques Cayouette.
} 


\section{Abstract}

This article focuses on the evolution of mycology in French Canada since the mid nineteenth century. Through an examination of this specific discipline, wider scientific practices will be considered, along with the key role played in French Canadian scientific development by Brother Marie-Victorin and the Brothers of the Christian Schools. As a result of the role that Marie-Victorin played in creating the Cercles des jeunes naturalistes and through his promotion of American and North American research, he fostered the career of many scientists, including René Pomerleau, who was important to the development of mycology in French Canada. However, though Pomerlean is generally credited with the creation of the two first amateur French Canadian mycological societies, he was not alone in fostering the study of fungi. The article focuses particularly on the development of the Cercles des mycologues amateurs de Montréal, which was founded in 1950.

Les années 1950 et 1951 marquent respectivement la fondation des deux premiers clubs de mycologie au Canada français, soit le Cercle des mycologues amateurs de Montréal (CMM) et le Cercle des mycologues amateurs de Québec (CMAQ). Cette similarité des noms de même que la simultanéité de leur création ne relèvent pas du hasard puisque René Pomerleau, considéré comme le père de la mycologie québécoise, aurait joué un rôle clé dans leur mise en place, du moins si l'on en croit ces deux organismes ${ }^{2}$, les sites officiels

\footnotetext{
${ }^{2}$ On consultera le résumé de l'histoire du Cercle de Montréal dans son site Web. L'auteur, Michel Famelart, a fait œuvre d'historien du club. Son texte, intitulé " Le Cercle des mycologues de Montréal : plus de quarante ans d'activité ", reprend un article qu'il fit paraître dans la revue Quatre-temps, vol. 17, n 3 (automne 1993), p. 51-52; voir l'onglet " $A$ propos du Cercle des mycologues de Montréal ", la rubrique "L'historique du CMM ", [En ligne], [www.mycomontreal.qc.cal structur.htm]. À propos du Cercle de Québec, voir sous l'onglet "Archives ", les diverses rubriques énumérées, [En ligne], [http://www.mycologiecmaq.org/index. php?option=com_content\&view=article\&id=39\&Itemid=39].
} 
gouvernementaux ${ }^{3}$ et même un ouvrage de synthèse sur l'histoire des sciences récemment rééditée. Professeur à l'Université Laval, Pomerleau donnait déjà, depuis quelques années, des cours de mycologie et, à titre d'invité du Jardin botanique depuis 1945, il venait régulièrement à Montréal pour offrir des cours pratiques sur les "champignons comestibles ", cours qu'il répéta à Québec en $1951^{6}$ et qui déboucha, là aussi, sur la création d'un club.

Non seulement Pomerleau aurait été le catalyseur de ces clubs, orientés à l'époque exclusivement vers la mycophagie, mais il aurait aussi participé à associer son nom à leur origine en racontant sa version de leur fondation. Ainsi, dès 1954, relate-t-il les trois premières années d'activités du club de Québec lors de l'assemblée de l'automne? Pour le Cercle de Montréal, il prononce, lors de son $25^{\mathrm{c}}$ anniversaire, une communication où il se donne le beau rôle ${ }^{8}$. Son indiscutable

${ }^{3}$ Le ministère des Ressources naturelles du Canada le présente comme le fondateur de ces deux clubs (voir [En ligne], [www.nrcan.gc.ca/com/deptmini/traipion/ renepomerleau-fra.php]). Ce commentaire fait sans doute écho à ce qu'écrivait André Fortin, ex-directeur de l'Institut de recherche en biologie végétale de l'Université Laval, au sujet de Pomerleau quand ce dernier a reçu le prix Marie-Victorin du gouvernement du Québec en 1981(voir [En ligne], [http://www.prixduquebec. gouv.qc.ca/recherche/desclaureat.asp?noLaureat=163]).

${ }^{4}$ Luc Chartrand, Raymond Duchesne et Yves Gingras, Histoire des sciences au Québec de la Nouvelle-France à aujourd'hui, nouvelle édition, Montréal, Éditions du Boréal, 2008 [1987], p. 353.

5 Jacques Rousseau, "Club des mycologues amateurs. Convocation ", 24 octobre 1950, dossier Samuel Brisson, Fonds Michel-Famelart déposé aux archives du Cercle des mycologues de Montréal (dorénavant ACMM-FMF).

${ }^{6}$ Voir "Cours sur les champignons comestibles", Le Soleil, août 1951, [En ligne], [http:// www. $m y$ cologie-cmaq.org/index.php?option=com_content\&view=article\&id=39 \&Itemid=39].

7 Voir dans le même site sous l'onglet "Archives ", la rubrique "Comptes rendus ", l'année 1954, [En ligne], [http://www.mycologie-cmaq.org/index.php?option=com_ content\&view=category\&layout=blog\&id=4\&Itemid=38].

${ }^{8}$ René Pomerleau, «Le quart de siècle du Cercle des Mycologues de Montréal ", Le Mycologue, vol. 1, n 3 (février 1977), p. 8-11. Cet article résume sa conférence livrée au Cercle des mycologues de Montréal à l'automne 1975. La version complète de ce texte est conservée sous forme de tapuscrit dans le Fonds Michel-Famelart (voir ACMM-FMF). 
renommée, révélée notamment par la publication, dès 1951, de son ouvrage d'identification Champignons de l'est du Canada et des ÉtatsUnis' ${ }^{9}$ qui servit d'ouvrage de référence pendant plus de deux décennies, fit le reste.

Nous cherchons à montrer que cette lecture des origines de ces deux cercles, ramenée au rôle prépondérant de Pomerleau, demeure tronquée. En effet, cette interprétation souffre d'un parti pris téléologique du fait que ce passé historique a été reconstruit à la seule lumière de ce héros de la mycologie ${ }^{10}$, René Pomerleau, qui devait forcément être à l'origine des premiers clubs. Même si le premier historien du Cercle de Montréal, Michel Famelart ${ }^{11}$, avait souligné le rôle tout aussi central du frère Rolland-Germain, compagnon d'excursions et de recherches du frère Marie-Victorin ${ }^{12}$, tous deux frères des écoles chrétiennes, il semble bien qu'on ait oublié le contexte qui a rendu possible cette fondation des premiers clubs de mycologie au Canada français. Au-delà des actions immédiates d'un Pomerleau qui relient les deux clubs, il y a d'abord et avant tout une société canadiennefrançaise qui a évolué sur le plan des mentalités, grâce au travail acharné d'un Marie-Victorin.

Parmi d'autres objectifs, nous voulons comprendre le rôle des Frères des écoles chrétiennes et du frère Marie-Victorin dont l'influence

9 Quoique cet ouvrage, paru aux éditions Chanteclerc en 1951, ait été à l'époque hautement considéré, ce n'est qu'en 1980, quand il publie son œuvre maîtresse Flore des champignons au Québec (Montréal, Les Éditions La Presse), que Pomerleau devint, aux yeux de la plupart des mycologues, "Monsieur mycologie québécoise".

${ }^{10}$ Selon André Fortin, qui participa à sa mise en candidature pour le concours du Prix du Québec en 1981, Pomerleau pouvait être considéré, à cette époque, comme un des cinq meilleurs mycologues au monde (voir [En ligne], [http:// www.prixduquebec.gouv.qc.ca/recherche/desclaureat. asp?noLaureat=163]). On pourra lire également une biographie relatant ses grands faits d'armes, publiée au lendemain de sa mort dans la revue Mycologia (Yolande Dalpé et T. Ginns, "René Pomerleau, 1904-1993 ", vol. 86, nº 4 (juillet-août 1994), p. 588-591).

${ }^{11}$ Voir les numéros consacrés aux $30^{\mathrm{e}}, 40^{\mathrm{e}}$ et $50^{\mathrm{e}}$ anniversaires du club, publiés dans Le Mycologue, vol. 6, $\mathrm{n}^{\circ} 1$ et $\mathrm{n}^{\circ \mathrm{s}} 2-3$ (1981); vol. 15, $\mathrm{n}^{\circ}$ spécial $40^{e}$ anniversaire (1990); et vol. $25, \mathrm{n}^{\circ}$ spécial $50^{\mathrm{e}}$ anniversaire (2000).

${ }^{12}$ Michel Famelart, "Le Cercle des mycologues de Montréal : quarante ans d'activité ", Le Mycologue, vol. 15 (novembre 1990), p. 11-12. 
sera examinée non pas sur l'ensemble des sciences comme il est coutume de le faire, mais à partir d'un des créneaux des sciences naturelles, la mycologie. Cet examen ciblé du rôle de Marie-Victorin éclaire ses interventions publiques en leur donnant une résonance spécifique. Par ailleurs, en tant que membre d'une congrégation religieuse considérée comme un ordre religieux mineur, Marie-Victorin nous force à nous interroger sur la place des grandes communautés religieuses enseignantes dans ces enjeux.

En filigrane, ce texte se veut une contribution à l'histoire de la mycologie au Canada français en relatant à grands traits l'état des sciences et des connaissances mycologiques jusqu'aux années 1950. Notre examen portera ensuite sur les premières décennies d'activités du Cercle de Montréal afin de voir comment il s'est distancié progressivement de Pomerleau.

\section{Des connaissances mycologiques embryonnaires}

Quand on cherche les premiers textes portant sur les champignons au Canada, il faut remonter au milieu du XIX ${ }^{e}$ siècle pour trouver quelques pages dans les ouvrages de botanique des pionniers. Il faut comprendre que, jusqu'à cette époque, ce sont les ouvrages européens qui servaient de référence aux botanistes et aux naturalistes de sorte qu'aucun inventaire sérieux des champignons d'ici n'avait encore été tenté. Un de ces pionniers est l'abbé Léon Provancher. La place qu'il leur réserve dans son volumineux ouvrage la Flore canadienne, paru en 1862, montre le peu d'intérêt qu'on leur porte alors. En effet, sur les quelque 800 pages, à peine dix décrivent quinze groupes de champignons répertoriés, et on compte seulement six illustrations regroupées sur une même page ${ }^{13}$. Ces pages offrent néanmoins une clé analytique qui permet grossièrement l'identification des grandes familles de champignons.

${ }^{13}$ Léon Provancher, Flore canadienne, Québec, Joseph Darveau imprimeur-éditeur, 1862 , p. 746 à 755 . 
Comme le rappelle Ralph Estey, les quelques efforts en mycologie sont alors tournés vers la mycologie appliquée, soit celle qui s'intéresse seulement aux répercussions économiques des maladies des plantes et des arbres ${ }^{14}$. Cette approche réductrice domine jusqu'aux années 1930. Il n'est donc pas étonnant de trouver fort peu de textes sur les champignons dans la principale revue de sciences naturelles du Canada français, Le Naturaliste canadien, dont le rédacteur n'est nul autre que l'abbé Provancher depuis sa fondation en 1868.

Heureux de présenter une liste de 25 champignons ${ }^{15}$ identifiés par un spécialiste européen, le baron Félix von Thümen, l'abbé y publie, en 1878, un court texte qui se veut un plaidoyer pour l'étude des champignons et des sciences naturelles.

Notre province possède probablement tout autant de champignons que la Grande-Bretagne, et non seulement nous n'avons ni listes, ni catalogues de ces productions végétales, mais pas même d'ouvrages dans nos bibliothèques, pour nous renseigner sur ces plantes [...] $[\mathrm{N}]$ ous avons parcouru en vain les plus grandes bibliothèques de Québec, toutes sont absolument muettes sur ce sujet, même celle de l'Université Laval avec ses 60000 volumes ${ }^{16}$.

Les nombreuses années que prendra Provancher à étudier l'entomologie d'ici explique peut-être l'absence de publications mycologiques de sa part dans la revue ${ }^{17}$. C'est pourquoi il faut encore attendre près de dix ans avant que cette publication ne revienne sur les champignons en publiant deux autres documents. Encore une fois, l'enjeu de ces textes est ici tout aussi révélateur du désert dans lequel se trouve la mycologie au Canada français. Racontons brièvement de quoi il

${ }^{14}$ Ralph H. Estey, Essays on the Early History of Plant Pathology and Mycology in Canada, Montréal, McGill-Queen's University Press, 1994, p. 247.

${ }^{15}$ On ne sera pas surpris de constater que presque tous les champignons mentionnés dans ce texte poussent sur des arbres, ce qui tend à montrer l'intérêt initial pour une approche économique de la mycologie.

${ }^{16}$ Léon Provancher, "Nos champignons ", Le Naturaliste canadien, vol. IX (janvier 1878), p. 7.

${ }^{17}$ On lira avec profit les quelques pages consacrées à Provancher dans l'Histoire des sciences au Québec, de Chartrand, Duchesne et Gingras, p. 189 et 194-197. 
s'agit. Revenant d'une excursion, l'abbé raconte sa découverte, au mois d'août 1884, d'un champignon remarquable, soit le Phallus impudicus $^{18}$. Prenant soin de le décrire avec minutie dans la revue, il reçoit par la suite une lettre d'un lecteur qui doute de son identification, car la description l'amène à penser qu'il s'agit d'une morille. Quelques mois plus tard, dans un autre texte ${ }^{19}$, l'abbé fera écho d'un échange de lettres avec ce lecteur et fera le point sur ces deux champignons. Une description comparative lui permet de conclure que l'identification initiale était correcte.

Cette pauvreté des connaissances en mycologie, jugement qu'il ne faudrait pas étendre à l'ensemble des connaissances scientifiques de cette époque, doit être mesurée d'abord à l'aune de textes comparables publiés par les contemporains anglophones de Provancher. À ce chapitre, mentionnons que, dès 1865 , D. A. Watt avait recensé 152 espèces de champignons poussant au Québec ${ }^{20}$, ce qui laisse sous-entendre une meilleure connaissance du domaine et un manque de communication entre les chercheurs. Cette impression est renforcée du fait que d'autres travaux publiés en anglais au tournant $\mathrm{du} \mathrm{xx}^{\mathrm{e}}$ siècle ne trouvent pas davantage écho dans les pages du Naturaliste canadien $^{21}$.

Il faut admettre néanmoins que, comme l'a montré l'historienne des sciences Mélanie Desmeules, Provancher entretient, entre 1875 et 1891, une correspondance avec le baron Félix von Thümen, qui

${ }^{18}$ Léon Provancher, "Un champignon remarquable ", Le Naturaliste canadien, vol. XVI (octobre 1886), p. 50-58.

${ }^{19}$ Léon Provancher, "Le Phallus et la Morille ", Le Naturaliste canadien, vol. XVI (février 1887), p. 115-119.

${ }^{20}$ D. A. Watt, "A Provisional Catalogue of Canadian Cryptogams ", Canadian Naturalist and Geologist, nouvelle série, vol. II (1865), p. 390-404.

${ }^{21}$ On compte plusieurs auteurs de publications mycologiques au Canada anglais à la fin du $\mathrm{XIX}^{\mathrm{e}}$ siècle et au cours des premières décennies du $\mathrm{xx}^{\mathrm{e}}$ siècle, tel le révérend Robert Campbell, qui avait identifié 129 espèces à partir de trois sites de cueillette au Québec ("Canadian Fungi ", The Canadian Record of Science, vol. IX (1903), p. 89-99). Voir, à ce sujet, Estey, Essays on the Early History of Plant Pathology, chapitre 9. 
est botaniste impérial à la cour d'Autriche ${ }^{22}$. De plus, l'abbé possède un herbier contenant plus de 300 espèces de champignons, qu'il n'a jamais rendu public. Si l'on doit lui reconnaître un intérêt marqué pour le monde des champignons, il faut cependant mentionner qu'il est relativement aisé de répertorier 300 espèces différentes de champignons sans nécessairement avoir à les identifier avec certitude. Retenons surtout que les connaissances en mycologie de l'abbé restent secrètes et sans lendemain. Par ailleurs, son décès et son remplacement à la direction du Naturaliste canadien expliquent peut-être l'absence de textes significatifs sur les champignons au Canada français pendant les quelques décennies suivantes.

\section{Le frère Marie-Victorin, grand défenseur des sciences au Canada français}

Qui n’a pas entendu parler du frère Marie-Victorin ${ }^{23}$, ce « symbole de la science au Canada français ${ }^{24}$ " auquel est rattaché le Jardin botanique, et de son célèbre ouvrage la Flore laurentienne? Cet ouvrage devient, à sa sortie en 1935 , le livre de référence en botanique. Ses interventions en faveur des sciences et des sciences naturelles en particulier sont toutefois moins connues du public.

Né en 1885, Conrad Kirouac joint, à l'âge de seize ans, l'ordre des Frères des écoles chrétiennes et prend le nom de Marie-Victorin. Bien qu'il ait pu fréquenter les grands collèges, il choisit de joindre l'ordre religieux chargé de son éducation à l'Académie commerciale de Québec. Cet ordre religieux - auquel on interdit d'enseigner le

${ }^{22}$ Mélanie Desmeules, "L'abbé Léon Provancher et l'étude des champignons ", La Corne d'abondance, vol. 21, no 1 (2005), p. 3-6.

${ }^{23}$ Parmi les nombreux textes et ouvrages consacrés à Marie-Victorin, deux doivent être soulignés, soit la longue biographie hagiographique rédigée par Robert Rumilly, qui est toujours indispensable (Le frère Marie-Victorin et son temps, Montréal, Les Frères des écoles chrétiennes, 1949), et la collection de textes de Marie-Victorin présentée par Yves Gingras (Frère Marie-Victorin, é.c., Science, culture et nation, textes choisis et présentés par Yves Gingras, Montréal, Éditions du Boréal, 1994).

${ }^{24}$ Yves Gingras, "L'itinéraire du Frère Marie-Victorin, é.c. (1885-1944) ", Revue d'histoire de l'Amérique française, vol. 39, n 1 (été 1985), p. 81. 
latin, réservé aux ordres majeurs offrant le cours classique comme les Jésuites et les Oblats - a son noviciat au Mont-de-la-Salle, à Maisonneuve, à l'endroit même où sera érigé, plus tard, le Jardin botanique. Privés de l'enseignement et de l'apprentissage du latin, les frères de cette communauté ont plus de temps à consacrer aux sciences $^{25}$ et une excellente réputation en pédagogie $e^{26}$. De plus, dans la première moitié $\mathrm{du} \mathrm{xx}^{e}$ siècle, cet ordre religieux s'est taillé une réputation internationale en botanique ${ }^{27}$, une science hautement valorisée chez eux.

Destiné par vocation à l'enseignement, Marie-Victorin est envoyé au collège de Longueuil où il enseignera de nombreuses années et où il fera la connaissance du frère Rolland-Germain. Ce dernier est envoyé de France au Canada en 1905, en raison de nouvelles lois sur l'éducation française - les lois Combes - qui non seulement interdisent le financement des écoles confessionnelles par l'État français, mais forcent également plusieurs congrégations religieuses à se séculariser ou à quitter le pays. Le frère Rolland-Germain deviendra son collaborateur le plus fidèle pendant près de quarante ans, et on peut même dire qu'au début de la carrière du jeune Kirouac, il est son maitre à penser $^{28}$.

Un premier rapprochement entre Marie-Victorin et la mycologie se manifeste sans doute dans la pratique des sciences naturelles telle qu'il la concevait, pratique qui ne peut se faire sans l'école de la route, c'est-à-dire sans un apprentissage sur le terrain. Comme il l'affirmait lui-même : "Quoi de plus hygiénique, de plus revigorant que ces courses au hasard des bois et des champs - la vraie méthode des

\footnotetext{
${ }^{25}$ Rumilly, Le frère Marie-Victorin et son temps, p. 6.

${ }^{26}$ Nive Voisine, Les Frères des écoles chrétiennes au Canada, t. 2 : Une ère de prospérité, 1880-1946, Sainte-Foy, Éditions Anne Sigier, 1991.

${ }^{27}$ Le frère Léon à Cuba, le frère Sennen en Espagne et le frère Héribaud-Joseph en France se sont taillé une place de choix au sein de la communauté internationale des botanistes (voir Rumilly, Le frère Marie-Victorin et son temps, p. 321).

${ }^{28}$ Ibid., p. 21.
} 
sciences naturelles $^{29}$. " Puisqu'il y a fort à parier que la présence d'un Pomerleau au sein des premiers clubs de mycologie soit directement ou indirectement redevable aux luttes menées par le frère MarieVictorin pour attirer de jeunes Canadiens-Français ${ }^{30}$ vers les sciences, mentionnons quelques-unes de ses interventions qui en disent long sur la mentalité de son époque, qui valorise principalement les humanités, soit la philosophie et la littérature, les professions libérales et une certaine France restée catholique.

À l'inverse du frère Rolland-Germain, Marie-Victorin n'est pas timide et n'a pas peur de prendre position sur la place publique. Grand gaillard, il ne craint pas de se faire des ennemis, tel le secrétaire de la province de Québec, Athanase David, qui, en raison de ses prises de position publiques, lui refusera une subvention ${ }^{31}$. Avec Lionel Groulx, Marie-Victorin est sans doute l'intellectuel du Canada français le plus inspiré et le plus marquant de la première moitié du $\mathrm{xx}^{\mathrm{e}}$ siècle ${ }^{32}$. Au déclenchement de la Première Guerre mondiale, les programmes de sciences offerts en français se limitent à celui de l'École polytechnique et à ceux nouvellement créés des écoles d'arpentage et de génie forestier. Dans les sciences pures, c'est le désert ${ }^{33}$.

Dès 1917, Marie-Victorin publie, à l'automne, le texte d'une longue conférence qu'il avait livrée à l'Université Laval au cours de l'été ${ }^{34}$ et dans laquelle il dénonçait le manque de scientifiques

${ }^{29}$ Frère Marie-Victorin, "Sciences naturelles au Canada : l'étude des sciences naturelles, son développement chez les Canadiens français ", La Revue canadienne, nouvelle série, volume XX (octobre 1917), p. 278.

${ }^{30}$ En conformité avec une pratique répandue en Ontario français, nous préférons écrire ce marqueur identitaire avec deux majuscules et un trait d'union mettant ainsi en évidence notre statut de peuple fondateur.

${ }^{31}$ Yves Gingras, "Science et communauté scientifique, 1910-1993 ", dans Robert Lahaise (dir.), Le Devoir, reflet du Québec au $20^{\circ}$ siècle, Montréal, Hurtubise HMH, 1994, p. 218.

${ }^{32}$ Frère Marie-Victorin, é.c., Science, culture et nation. Voir plus particulièrement le texte d'Yves Gingras, "Introduction : Marie-Victorin intellectuel », p. 7-29.

${ }^{33}$ Robert Lahaise, La fin d'un Québec traditionnel, 1914-1939, Montréal, L'Hexagone, 1994, p. 73.

${ }^{34}$ Frère Marie-Victorin, "Sciences naturelles au Canada : l'étude des sciences naturelles, son développement chez les Canadiens français ", La Revue canadienne, 
canadiens-français et d'intérêt pour les sciences : «Les empiètements, les envahissements de l'anglais sur le français dont nous nous plaignons dans le commerce, l'industrie et les services publics, ne sont rien à côté de ceux dont fatalement, et uniquement par notre incurable. indifférence, nous sommes affligés sur le terrain scientifique ${ }^{35}$. "Pas étonnant, croit-il, que des poètes et des écrivains canadiens-français fassent référence, dans leurs descriptions de la nature laurentienne, à des herbes et à des plantes européennes qui ne poussent pas ici à l'état sauvage. Platane, liane, thym, ajonc et bruyère, dit-il, sont étrangers à notre pays! En dernière partie, Marie-Victorin développe une critique fouillée de la Flore canadienne qui, malgré ses mérites, a beaucoup vieilli à ses yeux. Opposé à sa réédition, il affirme qu' " [i]1 nous faut une œuvre nouvelle, bâtie sur un plan moderne, et capable d'incorporer la totalité des découvertes et des études critiques du dernier demi-siècle ${ }^{36}$ ", commentaire qui annonce sa grande œuvre des années 1930.

Nommé, en 1920, titulaire de la chaire de botanique de la Faculté des sciences de l'Université de Montréal qui vient enfin d'être créée, Marie-Victorin ne tarde pas à se faire le grand défenseur des sciences naturelles en profitant de ses bons contacts au journal Le Devoir pour faire valoir ses idées ${ }^{37}$. Ainsi, dès 1922, il y publie un article faisant appel aux " littéraires qui, par la force des choses, ont jusqu'à ces dernières années, eu l'oreille et monopolisé l'attention comme les énergies de notre jeunesse de [...] hâter en autant qu'il est en eux, par leurs efforts désintéressés, l'avènement de notre peuple à la haute culture scientifique ${ }^{38}$ ". Cette culture scientifique, négligée par les

nouvelle série, volume XX (octobre 1917), p. 272-292, et novembre 1917, p. 339-358.

${ }^{35}$ Ibid., p. 275.

${ }^{36}$ Ibid., p. 358.

${ }^{37}$ Entre 1922 et 1944, il y signera près d'une quarantaine de textes importants (voir Gingras, "Science et communauté scientifique, 1910-1993 ", p. 218).

${ }^{38}$ Frère Marie-Victorin, "Vers la haute culture scientifique ", Le Devoir, 30 septembre 1922, p. 1. 
élites, est, selon lui, nécessaire, car " un peuple vaut non seulement par son développement économique, industriel ou commercial, mais encore et surtout par son élite de penseurs, de chercheurs et de savants, par son apport au capital scientifique de l'humanite ${ }^{39}$ ".

Passons rapidement sur son rôle dans la fondation de l'Association canadienne-française pour l'avancement des sciences (ACFAS) en $1923^{40}$, association dont il assume, au départ, la fonction de secrétaire général, tout comme le fait que, la même année, il participe aussi à la fondation de la Société canadienne d'histoire naturelle, un regroupement de naturalistes alors non représentés au sein de l'ACFAS. Ces nouveaux organismes montrent bien le regain d'intérêt pour les sciences au début des années 1920.

Deux ans plus tard, Marie-Victorin récidive dans Le Devoir en évoquant une rencontre au cours de laquelle son interlocuteur avait traité un professeur de botanique d'enseignant de "petites sciences", expression dont il cherche à montrer tout le ridicule, bien qu'elle rejoigne l'opinion de la plupart des élites bien-pensantes. Cherchant toujours à susciter les vocations en sciences, il dressera un réquisitoire qui dénonce, entre autres, l'enseignement des sciences par les premiers venus. Citons-en un extrait :

Nous ne serons une véritable nation que lorsque nous cesserons d'être à la merci des capitaux étrangers, des experts étrangers, des intellectuels étrangers; qu'à l'heure où nous serons maittres par la connaissance d'abord, par la possession physique ensuite des ressources de notre sol, de sa faune et de sa flore.

Pour cela, nous avons besoin de sérieuses vocations scientifiques, adéquatement cultivées. Il nous faut un plus grand nombre de physiciens et de chimistes, de biologistes et de géologues compétents qui soient autre chose que des fignoleurs se livrant à de faciles variations sur les travaux des autres [...] Nous avons assez de cette éternelle mouture d'enfantine vulgarisation de ces soi-

${ }^{39}$ Ibid.

${ }^{40}$ Voir, pour plus de détails, Yves Gingras, Pour l'avancement des sciences : histoire de l'ACFAS, 1923-1993, Montréal, Éditions du Boréal, 1994, le chapitre premier. 
disant synthèses brillantes, de ces jeux périscientifiques en dehors de toute observation directe $[\ldots]^{41}$.

Un dernier article de Marie-Victorin, publié dans Le Naturaliste canadien en 1930, doit être évoqué pour bien comprendre tout le travail qu'il a dû accomplir pour changer les mentalités de ses contemporains. Alors que Lionel Groulx avait cherché à montrer, dans son roman L'Appel de la race, les dangers de l'assimilation, et que l'élite canadienne-française dénonçait encore l'exode vers les États-Unis qui venait à peine de se terminer, Marie-Victorin, aussi nationaliste qu'il puisse être, souligne l'absolue nécessité de se rapprocher de l'Oncle Sam en recourant aux ouvrages et aux recherches américaines pour développer les sciences chez nous. Cet appel est particulièrement approprié en botanique - et, par ricochet, en mycologie :

$[D]$ ans nos universités canadiennes de langue française les services de Zoologie, de Botanique, de Géologie, doivent trouver leurs matériaux d'enseignement et leurs sujets de recherches dans le milieu naturel qui est la province de Québec et les provinces ou États environnants. Or, la province de Québec est une entité géographique purement artificielle dont la faune, la flore, les formations géologiques, les horizons paléontologiques débordent largement sur les États-Unis, sur l'Ouest canadien, quelques fois sur l'Asie, plus rarement sur l'Europe.

C'est dire que dans tous ces domaines, les objectifs nous sont communs, avec nos collègues des universités des États-Unis, et que notre bibliographie doit être surtout américaine et de langue anglaise.

Mais elle est puérile et niaise cette francolâtrie qui ne peut voir la science qu'à travers " l'article de Paris " qui en tout et pour tout, se

${ }^{41}$ Frère Marie-Victorin, «La province de Québec, pays à découvrir et à conquérir : à propos de culture scientifique et de libération économique ", Le Devoir, 26 septembre 1925, p. 1. 
tourne vers la Mecque parisienne et qui, coûte que coûte, cherche

à endosser un vêtement taillé à la mesure d'un autre ${ }^{42}$.

En conformité avec sa propre pratique, qui le conduit notamment à collaborer étroitement avec son collègue Merritt Lyndon Fernald de l'Université Harvard, Marie-Victorin adopte une position qui encouragera de nombreux chercheurs à poursuivre des études aux États-Unis, tels Émile Jacques, qui obtient son doctorat à l'Université Cornell en phytopathologie, et Jules Brunel, qui y fait un stage en cryptogamie, deux disciplines proches de la mycologie.

Nous ne disposons pas de preuve voulant que cette position ait pu influencer directement Pomerleau; néanmoins, il est possible de croire que le cheminement de Pomerleau ait été peu à peu marqué par Marie-Victorin. Jusqu'alors, Pomerleau avait suivi les étapes habituelles, allant en France poursuivre ses études en 1927 grâce à la loi des «bourses d'Europe " du gouvernement québécois. Il avait profité de « la francophilie militante de l'époque et, en particulier, [de] celle d'Athanase David, le secrétaire de la province chargé de l'application de cette $1 \mathrm{i}^{43}$ ", francophilie que dénonçait vertement Marie-Victorin.

À l'instar du programme québécois de bourses d'études qui reconnaît, au cours des années 1930, l'a poursuite des études dans les universités américaines, Pomerleau, qui rend régulièrement visite à Marie-Victorin ${ }^{44}$, se montre intéressé par les connaissances et les pratiques de notre voisin du Sud. Ainsi, dès 1932, est-il membre fondateur de la Mycological Society of America ${ }^{45}$, et ses recherches doctorales sur un parasite de l'orme, inconnu en Europe et en Asie, l'amènent tout naturellement à faire le point sur l'ensemble des connaissances nord-américaines, car le parasite est observé, comme

\footnotetext{
${ }^{42}$ Frère Marie-Victorin, "Les sciences naturelles dans l'enseignement supérieur ", Le Naturaliste canadien, vol. LVIII (novembre 1930), p. 227-228 et 230.

${ }^{43}$ Chartrand, Duchesne et Gingras, Histoire des sciences au Québec, p. 258.

${ }^{44}$ Rumilly, Le frère Marie-Victorin et son temps, p. 187.

${ }^{45}$ ACFAS, «Le Président de l'ACFAS pour 1951-1952: M. René Pomerleau, notes biographiques ", Annales de l'ACFAS, vol. 19 (1953), p. 49.
} 
il l'écrit lui-même, "sur toute l'étendue de l'aire de cet arbre, du Canada au Texas, de l'Atlantique aux Rocheuses ${ }^{46}{ }^{\prime}$. Tout en travaillant à la pépinière forestière de Berthierville, il accumule du matériel pour sa thèse de doctorat soutenue en 1937 à l'Ụniversité de Montréal.

\section{Deux institutions clés : les Cercles des jeunes naturalistes et le Jardin botanique}

En 1929, au retour d'un long voyage en Europe et en Afrique du Sud, Marie-Victorin rêve de doter Montréal d'un jardin botanique d'envergure internationale qui, à l'image du jardin de New York ou de celui de Berlin, inclurait un centre de recherches. Grâce notamment au soutien de son ami et disciple Jacques Rousseau et à l'appui d'un ancien étudiant du collège de Longueuil et maire de Montréal, Camilien Houde, ce rêve se réalisera après bien des embûches. Bien que fondé théoriquement en 1931, le Jardin ne sera officiellement ouvert qu'en $1939^{47}$, en raison d'un arrêt des travaux qui dure quatre ans, faute de soutiens financiers et politiques.

Si cette histoire est connue, on ignore souvent l'impact des Cercles des jeunes naturalistes sur la mycologie. Ici, la bougie d'allumage de ces regroupements de jeunes élèves est le frère Adrien, de la congrégation de Sainte-Croix, qui, depuis 1924, dirige deux cercles à l'école Beaudet. En janvier 1931, il propose à la Société canadienne d'histoire naturelle de créer d'autres clubs dans les différentes maisons d'enseignement. Réticent au départ, le frère Marie-Victorin se rallie à ce projet dont une des activités est la rédaction de ce qu'on appelle à l'époque des " tracts ", qui sont, en fait, de courts documents de vulgarisation destinés à être lus et discutés au sein des clubs.

${ }^{46}$ René Pomerleau, "Recherches sur le Gnomonia Ulmea (Schw.) Thüm. ", thèse de doctorat, Université de Montréal, 1937. Sa thèse sera publiée par tranches dans Le Naturaliste canadien à compter de novembre 1937; le passage cité est à la page 264 de ce numéro.

${ }^{47}$ Ainsi, ce n'est qu'en 1939 que l'Institut de botanique de l'Université de Montréal déménage au Jardin botanique. 
C'est Marie-Victorin lui-même qui rédigera, en 1932, le premier tract intitulé "Les Cercles des jeunes naturalistes ", dans lequel il explique qu' «il est possible de fonder un CJN dans toute école, petite ou grande, école de rang, collège, académie, couvent, institut agricole, école normale, juvénat, scolasticat, etc. ${ }^{48}$ ". En quelques années, des centaines de cercles sont fondés, davantage dans les institutions pour filles que dans celles pour garçons, de sorte qu'en 1940, on compte 462 clubs, dont les deux tiers sont féminins, et 13500 membres $^{49}$ :

L'Académie commerciale de Québec [en] est un foyer actif. Son directeur, le Frère Germain, est lui-même un collectionneur doublé d'un animateur. Au Collège de Longueuil, le Frère RollandGermain dirige le Cercle André Michaux, dont le Frère MarieVictorin a suggéré le nom et stimule le zèle. Au Collège Jean-deBrébeuf, le Père Taché a fondé le Cercle Huard, premier Cercle des Jeunes Naturalistes dans les maisons des Jésuites. Sous l'impulsion de Mère Marie-Élise, les institutions des Sœurs de Sainte-Anne sont entrées dans le mouvement. Une autre grande éducatrice, Mère Sainte-Alphonsine, formée à l'école de Mère Sainte-AnneMarie, lance les couvents de la Congrégation de Notre-Dame. L'élan n'est pas à créer, mais simplement à maintenir, chez les Sœurs de la Présentation-de-Marie, puisque deux élèves de leur École Normale de Saint-Hyacinthe se sont classées premières au concours du Devoir. Il se fonde aussi des Cercles dans les maisons des Sœurs de Sainte-Croix, des Sœurs du Bon-Pasteur, des Sœurs des Saints-Noms-de-Jésus-et-de-Marie ${ }^{50}$.

${ }^{48}$ Frère Marie-Victorin, "Les Cercles des jeunes naturalistes ", tract $n^{\circ} 1,30$ janvier 1932.

${ }^{49}$ Chartrand, Duchesne et Gingras, Histoire des sciences au Québec, p. 274. Comme le souligne avec justesse Pierrick Malissard, qui a étudié l'évolution de ces cercles, il faut être prudent dans l'évaluation du nombre de cercles et de membres, car les journaux de l'époque avaient tendance à gonfler les chiffres en rapportant fidèlement les données livrées par les responsables qui, ce faisant, voulaient accroître leur visibilité (voir son article, "Les cercles des jeunes naturalistes, ampleur et nature du mouvement, 1931-1971 ", Revue d'histoire de l'Amérique française, vol. 50, no 1 (été 1996), p. 9.

${ }^{50}$ Rumilly, Le frère Marie-Victorin et son temps, p. 195. 
Ces clubs constituent le terreau des futurs membres des clubs de mycologie. Certains responsables de ces cercles seront actifs au CMM, à l'instar du père Bernard Taché, qui y assumera plus tard un rôle central pendant plus de vingt ans. Quant à la présence de certaines congrégations religieuses féminines au CMM, elle plonge à coup sûr ses racines dans ces cercles qu'elles ont animés et fréquentés dès les années 1930.

Cela dit, malgré tous ces efforts, les sciences n'attirent encore guère de Canadiens-Français. Comme le montrait Jacques Rousseau dans un article publié en 1932, sur les 379 fonctionnaires fédéraux œuvrant au sein des services scientifiques, $97 \%$ sont canadiensanglais ${ }^{51}$. Certes, une discrimination envers les Canadiens-Français explique en partie cet écart entre les deux peuples fondateurs, mais le manque d'engouement pour ces disciplines y est aussi pour beaucoup.

Si l'on en croit quelques auteurs, l'enseignement des sciences demeure encore lamentable dans les collèges classiques au tournant des années 1930. Louis LeVasseur fait état de la place marginale de la science dans l'enseignement classique ${ }^{52}$. Jean-Francis Gervais et Jean Hénaire estiment que la période la plus sombre de l'enseignement des sciences dans ces institutions s'étire jusqu'en $1930^{53}$, tandis que pour Yves Gingras ${ }^{54}$, les collèges classiques dans leur ensemble affichaient, encore dans les années 1920, une résistance telle face à l'expansion des sciences que les responsables de l'ACFAS avaient alors

\footnotetext{
${ }^{51}$ Jacques Rousseau, "Les sciences pures chez les Canadiens français ", Opinion, vol. III, $\mathrm{n}^{\circ} 3$ (juillet 1932), p. 9-10.

${ }^{52}$ Louis LeVasseur, "L'enseignement dans les collèges classiques au XIX ${ }^{\mathrm{e}}$ siècle : une vision du monde en difficile harmonie avec la modernisation de la société québécoise ", Historical Studies in Education = Revue d'histoire de l'éducation, vol. $14, \mathrm{n}^{\circ} 1$ (2002), p. 58-59.

${ }^{53}$ Jean-Francis Gervais et Jean Hénaire, "L'enseignement des sciences dans les collèges classiques, $\mathrm{XIX}^{\mathrm{e}}$ et $\mathrm{XX}^{\mathrm{e}}$ siècles ", Recherches sociographiques, vol. $15, \mathrm{n}^{\circ} 1$ (janvier-avril 1974), p. 119-126.

${ }^{54}$ Gingras, Pour l'avancement des sciences, p. 43-48.
} 
ciblé les classes des écoles secondaires publiques pour faire avancer leur cause.

\section{3 : le premier congrès de l'ACFAS et le Mont-Saint- Louis}

À l'automne 1933, Marie-Victorin, Jacques Rousseau, cheville ouvrière de l'ACFAS, et le frère Alexandre des Frères des écoles chrétiennes et porte-parole de la Société canadienne d'histoire naturelle mettent sur pied deux événements qui se tiendront simultanément, soit le premier congrès de l'ACFAS, qui devient par la suite un événement annuel, et une exposition des Cercles des jeunes naturalistes. Ce dernier projet cherche à faire la promotion de la botanique et à clamer l'importance de maintenir ouverte l'Université de Montréal, très sérieusement menacée par la crise.

Les communications de l'ACFAS se tiennent à l'Université de Montréal tandis que l'exposition a lieu au Mont-Saint-Louis, institution phare des Frères des écoles chrétiennes où enseignent les plus brillants frères de la communautées. Cette exposition dure quinze jours et accueille, selon les porte-parole, 100000 visiteurs, grâce à l'implication de 137 cercles, dont 97 étaient féminins. Herbiers et collections de toutes sortes y sont exposés, sans que l'on sache si la mycologie y trouvait une place.

Parmi les conférences de l'ACFAS, certaines touchent néanmoins la mycologie comme celles de Pomerleau. Il livre, à ce premier congrès de 1933, sept communications dont quelques-unes touchent différentes dimensions des maladies des arbres provoquées par des champignons ${ }^{56}$. Comme il l'avoua lui-même, sa formation en phytopathologie tant à Sainte-Anne-de-la-Pocatière qu'au collège MacDonald, où il

\footnotetext{
${ }^{55}$ Le cours scientifique qu'on y offre permet, par exemple, d'entrer directement en $2^{\mathrm{e}}$ année à l'École polytechnique de Montréal. Au sujet de l'excellence de son corps professoral, voir Guy Samson, Bernard Lamarre, le génie d'une vie, Montréal, Presses internationales Polytechnique, 2007, p. 25.

${ }^{56}$ Pour les titres et un résumé de ses communications, voir les Annales de l'ACFAS, Montréal, 1934, p. 71-73.
} 
obtint sa maîtrise, ne lui avait fourni aucune connaissance sur les espèces charnues qu'il voyait pourtant dans les champs. C'est pourquoi les recherches et les communications sont encore cantonnées à la mycologie appliquée, ignorant du coup ces champignons charnus, comestibles ou vénéneux. La connaissance de ces derniers se fera véritablement en marge de sa formation en France à la fin des années $1920^{57}$, quoiqu'elle reste avant tout une pratique personnelle encore dépourvue de résonance scientifique.

En fait, c'est seulement à la fin des années 1930 que les champignons charnus font l'objet d'une démarche scientifique ou sont mentionnés dans certaines publications. En effet, l'année 1938 marque un tournant important pour la mycologie au Canada français. Ayant participé l'année précédente, pour la première fois, au congrès de la Société américaine de mycologie à Hanover au New Hampshire, Pomerleau invite les membres à Duchesnay, au Québec, pour l'année suivante. La rencontre, qui a lieu à la fin août, connaît un immense succès avec 836 espèces répertoriées grâce à la participation de nombreux spécialistes en mycologie de l'Amérique du Nord, parmi lesquels figure son collègue de l'Université de Montréal, Jules Brunel.

C'est avec ce dernier qu'il entreprend, la même année, la publication d'un inventaire des champignons du Québec dans Le Naturaliste canadien. Cet inventaire, qui occupe plusieurs numéros de la revue jusqu'au début des années $1940^{58}$, ne présente tout de même que 36 champignons, tous des polypores, dont les descriptions sont toutes signées par Pomerleau. Peut-être la guerre met-elle un terme à ce projet dont Brunel semble absent. Pourtant, c'est Brunel et non Pomerleau qui est alors professeur de mycologie, comme en fait foi l'appartenance institutionnelle mentionnée en tête de l'article. Précisons que Pomerleau sera engagé à l'Université Laval en 1940, pour

\footnotetext{
${ }^{57}$ Pomerleau, "Le quart de siècle du Cercle des mycologues de Montréal ", tapuscrit, p. 4, ACMM-FMF.

${ }^{58}$ Voir vol. 65 (1938), p. 5-12, 98-102, 138-140; vol. 66 (1939), p. 28-32, 90-94, $123-128,195-196,223-228$; vol. 67 (1940), p. 24-30, 91-96 et 229-232.
} 
être titulaire du cours de mycologie et de phytopathologie trois ans plus tard ${ }^{59}$. Cela dit, c'est Brunel qui, en 1933 et en 1939, rédige deux tracts sur les champignons pour les Cercles des jeunes naturalistes $^{60}$. C'est encore lui qui, à deux reprises entre 1941 et 1943, fera état des secrets des champignons à Radio-Collège, dans le cadre d'une série radiophonique de vulgarisation scientifique ${ }^{61}$.

Commencés en 1945, les cours d'identification des champignons profitent assurément d'une société des loisirs qui se met en place après les privations de la guerre. Ils profitent aussi de la grande prospérité de cette période appelée "Les Trente Glorieuses ». Si la filière des Cercles des jeunes naturalistes demeure un fil conducteur déterminant des origines des cercles des mycologues, cela ne doit pas nous faire perdre de vue que la plupart des gens se méfient alors souverainement des champignons et que s'y intéresser, en 1950, demeure un geste de culture.

René Pomerleau ne résume pas à lui seul la mycologie canadiennefrançaise, bien qu'il faille reconnaitre son rôle de catalyseur dans la formation des deux premiers cercles de mycologues. Au tournant des années 1940, Jules Brunel est tout aussi présent sur l'échiquier mycologique $^{62}$. Et, quand on parcourt la liste des communications présentées à l'ACFAS entre la fin des années 1930 et la date de fondation du CMM, force est de reconnaitre le nom de quelques autres chercheurs intéressés par les champignons, comme Émile Jacques ${ }^{63}$ dont on

${ }^{59}$ ACFAS, "Le Président de l'ACFAS pour 1951-1952 : M. René Pomerleau », p. 49.

${ }^{60}$ Jules Brunel, "Le monde mystérieux des champignons ", Cercle des jeunes naturalistes, tract $n^{\circ}$ 6, 20 mars 1933; "La vie méconnue des champignons ", tract $\mathrm{n}^{\circ}$ 64, 15 février 1939.

${ }^{61}$ Rumilly, Le frère Marie-Victorin et son temps, p. 375 et 388.

${ }^{62}$ En novembre 1969, il a prononcé, au Cercle de Montréal, une communication intitulée "Une exposition d'ouvrages récents et anciens sur la mycologie ", et il deviendra ensuite, pour quelque temps, membre en règle du Cercle (voir Florence Montreuil, "Les livres comptables du Cercle ", ACMM).

63 "Émile Jacques, août 1932 ", Archives de l'Université de Montréal, E01181FP0110-1. Ajoutons qu'au Congrès de l'ACFAS de 1946, il prononce une conférence intitulée "Quelques champignons du lac Mistassini " (voir les Annales de l'ACFAS, 1947, p. 11). 
trouve, dans les archives de l'Université de Montréal, une photographie datée de 1932 où il tient deux beaux champignons que l'on peut aisément identifier comme étant des amanites.

Ces quelques personnes qui s'intéressent à la mycologie sont néanmoins toutes tributaires et disciples du frère Marie-Victorin et ont profité du changement de mentalité qu'il a, avec d'autres propagandistes des sciences, insufflé aux Canadiens-Français de son époque. Ainsi, il a sans doute contribué à rendre acceptable le fait qu'ils se tournent vers les institutions et les pratiques scientifiques américaines, sans que leur patriotisme soit mis en cause. La mort de Marie-Victorin, en 1944, va forcer ses proches collaborateurs à assumer de nouvelles fonctions. C'est ainsi que Jacques Rousseau devient directeur du Jardin botanique tandis que Jules Brunel assure dorénavant la direction de l'Institut botanique, ce qui l'oblige peut-être à s'éloigner de la mycologie.

\section{La fondation du Cercle des mycologues de Montréal ${ }^{64}$}

Attardons-nous maintenant à la fondation du Cercle de Montréal, en récapitulant d'abord les principales étapes des trente premières années. Ainsi, le 31 octobre 1950, au Mont-Saint-Louis, rue Sherbrooke, se tenait la deuxième réunion du Cercle des mycologues amateurs de Montréal, présidée par le frère Rolland-Germain des Frères des écoles chrétiennes et dont le secrétaire est Jacques Rousseau, directeur du Jardin botanique. Parmi les personnes présentes aux réunions de l'automne 1950, on compte quelques autres frères des écoles chrétiennes et des religieuses de deux congrégations, soit les Sœurs des Saints-Noms-de-Jésus-et-de-Marie et les Sœurs de Sainte-Croix. La grande majorité est laïque, dont un

${ }^{64}$ Cette section de l'article a été en partie publiée dans le bulletin du Cercle des mycologues de Montréal sous le titre « La fondation du Cercle des mycologues de Montréal et les récits historiques de ses trois premières décennies d'existence ", Le Mycologue, vol. 35, $\mathrm{n}^{\circ}$ spécial $60^{\circ}$ anniversaire (novembre 2010), p. 31-46. Nous remercions sa rédactrice de nous avoir autorisé à le reprendre sous une nouvelle perspective. 
bon nombre de femmes. Afin de recruter d'autres membres, on y évoque l'idée de consulter la liste des personnes qui ont suivi les cours de mycologie de René Pomerleau.

Enseignant à la retraite depuis 1946, le frère Rolland-Germain réside au Mont-Saint-Louis. C'est là où, après la guerre, se rencontrent des amis et des connaissances qui partagent avec lui une passion commune pour les champignons et qui organisent, à l'occasion, des excursions. On connaît les noms de onze d'entre eux qui, en janvier 1950, signent leur nom sur une page qui a été conservée, en déclarant être les fondateurs du Cercle.

Jacques Rousseau fait partie de ce groupe d'amateurs de bons champignons. Ami de René Pomerleau, il a invité ce dernier, depuis peu professeur de mycologie à l'Université Laval, à donner des cours pratiques. À la fin des cours, en septembre 1950, l'idée de prolonger et de formaliser les activités du groupe initial amène Rousseau à convoquer les gens inscrits et toutes les personnes intéressées à « la formation d'un club ou société de mycologues amateurs s'occupant de la cueillette des champignons comestibles ${ }^{65}$ ".

Au cours de l'automne, quelques rencontres au Mont-Saint-Louis permettent de se doter d'un conseil d'administration et de règles de fonctionnement, comme celle de la tenue d'une assemblée générale annuelle, dont la première est prévue pour le samedi 20 janvier 1951 dans cette même institution. Parmi les personnes assistant à ces rencontres dont les noms ont été consignés dans les archives du Cercle, on retrouve neuf des onze membres fondateurs. S'y joignent d'autres personnes parmi lesquelles plusieurs avaient sans doute suivi les cours de Pomerleau, dont un certain nombre de religieuses de différentes communautés.

Le frère Rolland-Germain en est le premier président, fonction qu'il assume pendant cinq ans et qui explique que le port d'attache institutionnel du Cercle demeure le Mont-Saint-Louis, dont l'empla-

${ }^{65}$ Jacques Rousseau, "Club des mycologues amateurs. Convocation ", 24 octobre 1950, dossier Samuel Brisson, ACMM-FMF. 
cement central, dans le quartier latin de la bourgeoisie canadiennefrançaise, constitue un atout. En raison de ses vastes connaissances et fort du prestige de son grand ami Marie-Victorin, on avait besoin de lui pour identifier les champignons et pour ajouter de la crédibilité au club. Quant à Jacques Rousseau, il n’aura été qu'un simple initiateur, car très vite, dès la fin de l'automne 1950, il cède sa fonction de secrétaire, trop occupé qu'il est au Jardin botanique. D'autres personnes se joignent au frère pour former la première équipe du Cercle.

Si le Cercle se donne comme objectif principal des « excursions qui permettent aux chercheurs de champignons l'étude sur place ${ }^{66}$ ", activités dont le succès ne se démentira pas par la suite, il y a également la tenue de conférences qui s'impose dès la première année. Le premier conférencier est Pomerleau : " [À] l'occasion de la publication de l'ouvrage du Docteur Pomerleau, il s'agirait d'une réunion avec conférence et exposition des aquarelles de M. Jackson qui ont servi pour l'illustration du livre ${ }^{67}$. "Ce livre de vulgarisation ${ }^{68}$ contribue $^{2}$ certainement à faire la promotion des champignons et, par ricochet, celle des activités du club, tout en développant aussi une expertise chez les membres.

\section{Le Cercle devient l'affaire du père Taché}

On ne sait pas précisément pourquoi le frère Rolland-Germain quitte la présidence en 1955, date où il reçoit un doctorat honorifique de l'Université de Montréal. Âgé de près de soixante-quinze ans, il ne se sent peut-être plus aussi alerte. Effacé toute sa vie derrière MarieVictorin, on ne s'étonnera pas qu'il quitte discrètement la direction $\mathrm{du}$ Cercle sans laisser de traces. Si les réunions se tiennent au Mont-

\footnotetext{
${ }^{66}$ " Procès-verbal de l'Assemblée du 2 décembre 1950 ", dossier Samuel Brisson, ACMM-FMF, p. 1.

${ }^{67}$ Ibid., p. 2. Comme on l'a vu plus haut, c'est en 1951 que Pomerleau publie Champignons de l'est du Canada et des Etats-Unis grâce à l'intervention de Rousseau.

${ }^{68}$ Pomerleau, "Le quart de siècle du Cercle des mycologues de Montréal ", p. 9.
} 
Saint-Louis pendant quelque temps encore, c'est peut-être parce qu'il continue néanmoins à identifier des champignons les lundis de septembre et d'octobre ${ }^{69}$. C'est le jésuite Bernard Taché qui assumera progressivement la gestion quotidienne et le leadership du Cercle. Il peut compter sur la trésorière Francine Montreuil de sorte que même si les présidents et les conseillers passent, ce tandem parvient à assurer la continuité du Cercle au cours des vingt années suivantes.

Comme on l'a vu plus haut, le père Taché, disciple de MarieVictorin, est le premier jésuite à avoir adhéré, au cours des années 1930, aux Cercles des jeunes naturalistes. Celui que ses élèves appellent le «Père Bibitte " n'est pas très représentatif de sa communauté dont l'enseignement reste tourné vers les humanités : « Les CJN, rappelle Malissard, ne furent jamais très nombreux dans les collèges classiques $^{70}$. » Dès 1953, le père Taché assume le poste de vice-président du club et, à compter de 1955, et ce, pendant de nombreuses années, il se charge du secrétariat, tout en faisant profiter les membres de ses connaissances mycologiques, comme l'atteste une convocation à une réunion du 6 juin $1955^{71}$. Si l'on en juge par les livres de comptes laissés par madame Montreuil, il déménage, en 1957, le secrétariat du Club du Mont-Saint-Louis au laboratoire de biologie du collège Sainte-Marie, où il exerce son enseignement.

L'examen de ces livres de comptes nous permet aussi d'estimer le nombre de membres à une quarantaine de personnes au maximum; sur ce plan, Famelart a sans doute exagéré en l'estimant à une centaine $^{72}$. Soyons plus prudents : leurs activités n'attirent qu'une poignée de participants dont plusieurs sont médecins et membres

\footnotetext{
${ }^{69}$ Michel Famelart, "Le Cercle en vitesse de croisière : vingt ans d'activités ", Le Mycologue, vol. 25 (juillet 2000), p. 17.

${ }^{70}$ Malissard, "Les cercles des jeunes naturalistes ", p. 22.

${ }^{71}$ Père Bernard Taché, convocation du 6 juin 1955 aux membres du Cercle des mycologues amateurs, ACMM. Ses connaissances dans le domaine de la mycologie sont encore bien imparfaites en 1955, si l'on en juge par la demande qu'il adresse aux membres d'apporter, pour cette réunion du début juin, des spécimens frais de russules (ce qui est très tôt pour cette variété de champignons).

${ }^{72}$ Famelart, "Le Cercle en vitesse de croisière : vingt ans d'activités ", p. 21.
} 
du clergé. Selon nous, cette faible participation se poursuit jusqu’à la fin des années 1960.

Ce n'est qu'au tournant des années 1970 que le nombre de personnes qui paient leur cotisation dépasse la centaine. Et dès 1972, on atteint presque 200 membres, bien que l'on ne sache pas combien d'entre eux ont payé leur cotisation. Plusieurs facteurs expliquent cette croissance rapide parmi lesquels figurent la nouveauté que représentent les champignons pour le grand public, les effets de la démocratisation de l'enseignement, qui profite à la nouvelle génération des baby-boomers, une plus grande ouverture sur le monde provoquée par EXPO 67 et un goût plus marqué pour les pratiques de plein air, qui sont davantage en harmonie avec les idées contestataires de la contre-culture.

Cette popularité croissante du Cercle attire de nouveaux venus et de nouvelles compétences qui permettent de renouveler progressivement la gestion du Cercle, tout en posant aussi de nouveaux défis. Son mode de fonctionnement très informel - ce qui explique d'ailleurs les carences des archives pour les premières décennies - n'allait plus suffire. C'est pourquoi le nouveau président du club, Jean Beaudry, professeur de botanique à l'Université de Montréal, cherche, à la même époque, à faire adopter une constitution. Beaudry est l'archétype d'une nouvelle génération de membres qui s'implique au conseil d'administration, soit les professeurs et les étudiants du Département de biologie de l'Université de Montréal qui remplacent, au cours des années 1970, les médecins qui, jusqu'alors, siégeaient au conseil.

Les annotations de l'abbé J.-Albert Potvin au projet de modification de la constitution permettent également d'éclairer une autre dimension qui est en train de bouleverser radicalement le Cercle. Larticle 2 du projet de constitution précise que " [l]e but du Cercle est de favoriser l'étude et la connaissance des champignons et la mycophagie, par tous les moyens appropriés et tout particulièrement par le moyen d'excursions, de conférences, causeries, séances de 
projection de films, visites, etc. à caractère mycologique ${ }^{73} »$. En plus de suggérer de nombreuses modifications stylistiques au texte, l'abbé propose deux changements qui soulignent sa volonté de réorienter le club. En effet, en tant que naturaliste, il propose d'éliminer la référence à la mycophagie pour mettre davantage l'accent sur « l'étude et l'amour de la nature, en insistant spécialement sur la connaissance des champignons $[\ldots]^{74}{ }^{7}$. Ce souhait s'accompagne d'une autre proposition, soit celle de modifier le nom même du Cercle qui perdrait officiellement l'épithète d'amateur. Peu importe que ces modifications n'aient pu être mises en vigueur ${ }^{75}$, elles annoncent, d'ores et déjà, un important changement de mentalité au sein du Cercle.

Parallèlement à cette volonté de transformer le club, l'équipe qui monopolise la gestion du Cercle depuis près de vingt ans montre des signes de vieillissement et ne semble pas disposée à modifier son fonctionnement. Ainsi, le père Taché, qui a pris sa retraite de l'enseignement en $1969^{76}$, craint-il les idées farfelues des nouveaux venus ${ }^{77}$. Le Cercle demeure SON affaire. D'ailleurs, à sa mort, la notice nécrologique rédigée par sa communauté mentionne qu'il en est le fondateur ${ }^{78}$ !

Tout se passe comme si le père Taché avait pu faire avorter un projet de constitution qui avait l'assentiment du président et du vice-président ${ }^{79}$. Dans le récit historique de cet épisode que Famelart

${ }^{73}$ Projet de constitution, annexé à une lettre de l'abbé Potvin envoyée à Jean R. Beaudry, le 5 février 1970, ACMM-FMF.

${ }^{74}$ Ibid.

${ }^{75}$ Famelart, "Le Cercle en vitesse de croisière : vingt ans d'activités ", p. 22.

${ }^{76}$ Les Jésuites ont dû alors quitter le collège Sainte-Marie, devenu une constituante de la nouvelle Université du Québec à Montréal. Une partie des rencontres et des activités du Cercle se tiennent dorénavant ailleurs et notamment au Jardin botanique.

${ }_{77}$ Famelart, "Le Cercle des mycologues de Montréal : quarante ans d'activité », p. 29.

${ }^{78}$ Alfred Ducharme, s.j., "Le Père Bernard Taché, S.J. (1902-1984) ", notice nécrologique parue dans Anciens de Sainte-Marie : bulletin de liaison, vol. 42, $\mathrm{n}^{\circ} 3$ (octobre 1984), p. 8.

${ }^{79} \mathrm{La}$ consultation du Fonds Bernard-Taché aux archives des Jésuites aurait pu nous éclairer à ce sujet, mais il ne sera accessible que trente ans après son décès, soit en 2014. 
livrera plus tard, ce dernier a préféré ne pas trop incriminer Taché, peut-être parce qu'il lui était resté attaché en tant qu'ancien étudiant. Mais là où Jean Beaudry et l'abbé Potvin avaient échoué en 1970, d'autres allaient bientôt réussir.

\section{Le Cercle s'incorpore et accroît sa visibilité}

N'apparaissant pas sur la liste des membres de 1972, Louis Richard, professeur de mathématiques au cégep Ahuntsic, s'impose dès l'année suivante. Passionné de mycologie, il se fait remarquer, dès l'automne 1973, en rédigeant et en diffusant trois communiqués scientifiques. Inspirant confiance au père Taché, il représente le Cercle à la nouvelle Fédération québécoise des loisirs scientifiques en 1974 et décroche, la même année, une petite subvention qui, combinée à un versement de l'ACFAS, permet de financer la publication de textes de vulgarisation intitulés Documents mycologiques. Il est l'initiateur et le seul rédacteur de ces cinq documents qui paraissent entre janvier 1975 et l'hiver 1976. Richard avouera lui-même qu'ils étaient " trop scientifiques, trop difficiles pour la majorité des membres ${ }^{80}$ ».

Trouver le juste ton pour rejoindre le plus de membres possible n'était visiblement pas simple :

Il faudrait, dit-il en avril 1976, que nous lancions quelque chose de plus simple, de plus accessible à tous : une petite revue, un bulletin, qui prendrait la relève des Documents Mycologiques. Ce serait notre lien véritable, notre journal, où nous pourrions inscrire les potins du Cercle, les listes d'excursions, le relevé des espèces trouvées lors de ces promenades, la description détaillée des espèces nouvelles $[\ldots]^{81}$.

En août 1976, il lance, à titre de responsable du comité de rédaction, Le Mycologue, encore publié aujourd'hui.

${ }^{80}$ Louis Richard, "Rapport du président pour l'assemblée générale du Cercle des mycologues de Montréal, le 12 avril 1976 ", procès-verbal de la $15^{\mathrm{e}}$ réunion du Conseil d'administration, 12 avril 1976, p. 11.

${ }^{81}$ Ibid., p. 12. 
Le dynamisme de Richard, sans doute dérangeant, conjugué à l'attaque de paralysie du père Taché en janvier 1975, à la démission de madame Montreuil en février et à la volonté de plusieurs membres de revoir l'avenir du Cercle allaient aboutir à une restructuration majeure. Son incorporation, dont le dossier est mené par Famelart en 1975, suivie de l'adoption de règlements généraux allaient conduire à plusieurs changements, à commencer par un nouveau nom souhaité depuis plusieurs années - puisqu' on laisse finalement tomber l'épithète " amateur ". Le consensus a aussi été facile à atteindre quant à la redéfinition des objectifs du Cercle : faire la promotion de «l'étude et la connaissance des champignons, plus particulièrement des champignons supérieurs".

Ainsi, à partir de 1975, la gestion du Cercle est-elle beaucoup mieux structurée, ce qui facilite aujourd'hui le travail des historiens et des archivistes. Dorénavant sont conservés les procès-verbaux des réunions du conseil d'administration, comme l'oblige la loi régissant les corporations. Bien que le président Richard innove à nouveau, en 1976, en offrant les premiers cours d'initiation à la mycologie ouverts au grand public, ses décisions unilatérales dérangent plusieurs membres du conseil d'administration, qui croient davantage au travail collectif. N'en disons pas plus, il démissionne de son poste le 31 octobre 1977.

Plus importante est cette arrivée au conseil d'administration de diplômés universitaires en biologie, qui permettent au club de parfaire son expertise en mycologie grâce à diverses initiatives ${ }^{82}$, notamment l'embauche par le Cercle du mycologue Yves Lamoureux, le démarrage d'un herbier mycologique et d'un inventaire des champignons de la région montréalaise, etc. Déjà à la fin des années 1990, 1700 espèces de champignons sont répertoriées et environ 300 d'entre elles sont des ajouts aux travaux de Pomerleau ${ }^{83}$. Ces activités scientifiques

\footnotetext{
${ }^{82}$ Ces diverses initiatives sont citées dans le bref historique du calendrier-souvenir du 60 anniversaire du Cercle paru en 2010.

${ }^{83}$ Matthieu Sicard et Yves Lamoureux, Connaître, cueillir et cuisiner les champignons sauvages du Québec, Montréal, Fides, 1999, p. 11.
} 
débouchent sur une série de publications savantes ${ }^{84}$ qui marquent une appropriation de connaissances ${ }^{85}$ et qui font oublier ce qui était considéré jusqu’alors comme la bible du mycologue québécois, soit le volumineux ouvrage de Pomerleau publié en 1980, Flore des champignons au Québec.

Nous formulons l'hypothèse qu'il existe un lien entre cette quête de connaissances mycologiques et l'utilisation que fera le Cercle du nom de Pomerleau. Jusqu'alors, le club avait reconnu, dans son histoire officielle diffusée en quatrième de couverture du Mycologue, Pomerleau comme son fondateur, ce qui lui permettait de profiter de son prestige et de se donner des lettres de noblesse. Au lendemain $\mathrm{du} 50^{\circ}$ anniversaire du club, célébré en 2000, le Cercle prend finalement ses distances vis-à-vis de lui, pour l'exclure de son récit fondateur et reconnaitre en lieu et place la contribution du frère RollandGermain. Tout se passe comme si, ayant longtemps servi de caution scientifique, Pomerleau était devenu inutile. Ce geste, facilité par le décès de ce dernier en 1993, n'aurait pu se faire sans les recherches mycologiques des membres du Cercle.

\section{Conclusion}

En fin de course, quelques éléments doivent être soulignés. Ainsi, une étape importante de la mycologie est-elle atteinte à la fin des

${ }^{84}$ Outre l'ouvrage cité à la note précédente, mentionnons le cédérom produit en 2002 par le Cercle et intitulé Mille et un champignons du Québec dont les auteurs sont Jean Després, Yves Lamoureux, Raymond Boyer, Raymond Archambault et André Jean. Six autres ouvrages doivent aussi être signalés:Yves Lamoureux et Jean Després, Champignons du Québec, t. I : Les bolets (Montréal, CMM, 1997); Yves Lamoureux, Champignons du Québec, t. II : Les Amanites (Montréal, CMM, 2006); Raymond McNeil, Le grand livre des champignons du Québec et de l'est du Canada (Waterloo, Éditions Michel Quintin, 2006) et Champignons communs du Québec et de l'est du Canada (Waterloo, Éditions Michel Quintin, 2010); Jean Després, Champignons comestibles du Québec (Waterloo, Éditions Michel Quintin, 2008) et Clef des groupes de champignons du Québec (Montréal, CMM, 2010).

${ }^{85}$ Cette appropriation de connaissances est également visible sur la Toile. En effet, plusieurs membres du Cercle, notamment Renée Lebeuf, participent régulièrement à l'enrichissement de cet inventaire dans Internet ([En ligne], [mycoquebec.org]). 
années 1930 alors que l'intérêt pour une mycologie appliquée cède la place à une connaissance en soi des champignons. Profitant d'une large diffusion des sciences, grâce aux congrès de l'ACFAS, aux Cercles des jeunes naturalistes et aux expositions scientifiques, les champignons deviennent, comme d'autres segments de la nature, des objets de curiosité pour les uns et des objets de recherche pour d'autres. La guerre viendra interrompre ce premier apprivoisement collectif des champignons, qui reprendra de plus belle dès le conflit terminé.

Force est de reconnaître néanmoins une très faible participation des couches populaires aux activités d'un club de mycologie. Il convient sans doute de rappeler que la plupart des membres font partie de la petite bourgeoisie canadienne-française - on compte alors peu d'anglophones et d'immigrants. Ces membres font certes partie d'une minorité puisque cueillir et consommer des champignons étaient et demeurent des gestes de culture distinctifs. Que ce soit pour devenir membre des Cercles des jeunes naturalistes ou du Cercle de Montréal, l'accès à une éducation secondaire qui, même publique, demeure longtemps le privilège d'une minorité, s'est révélé essentiel pour élargir ses horizons et faire fi des préjugés tenaces transmis de génération en génération contre tous les champignons. $\mathrm{Si}$, au tournant des années 1970 , il y a augmentation rapide des effectifs, la raison première demeure probablement un plus grand accès à une éducation publique gratuite.

Si l'on ne connaît pas les motifs du départ des médecins du conseil d'administration du Cercle, il ne fait pas de doute que l'arrivée des biologistes demeure, comme on vient de le voir, lourde de conséquences. Mais il ne faudrait pas croire que cette nouvelle génération de membres provoque une rupturé avec le passé et que les chercheurs amateurs ont laissé la place à des professionnels. Comme on l'a vu dans les publications scientifiques du club, un Jean Després, ou une Renée Lebeuf sur la Toile - qui n'ont pourtant pas de formation en botanique ni en biologie - contribuent et ont largement contribué à cette quête de connaissances mycologiques, ce qui n'est pas d'ailleurs sans rappeler le rôle d'un pionnier comme Louis Richard. 
En terminant, une hypothèse émise par des collègues sociologues, il y a une trentaine d'années, mérite d'être partiellement reprise. Au Canada français, les sciences naturelles auraient proportionnellement attiré davantage les personnes provenant de milieux modestes ${ }^{86}$. Cette attirance des petites gens pour les " petites sciences ${ }^{87}$ » s'expliquerait par le fait que ce sont certaines communautés religieuses, plus ouvertes aux sciences, comme les Frères des écoles chrétiennes ${ }^{88}$, qui dirigent alors les institutions d'enseignement qui leur sont plus accessibles, soit des écoles d'enseignement secondaire public, des écoles normales et des académies commerciales. Ces dernières sont certes moins prestigieuses que les collèges classiques, qui n'ont pas toutes un père Taché pour faire oublier leur engouement pour les humanités, mais elles ne perdent pas de temps à faire de la place aux sciences et aux Cercles des jeunes naturalistes.

\footnotetext{
${ }^{86}$ Francine Descarries-Bélanger, Marcel Fournier et Louis Maheu, « Le frère MarieVictorin et "Les Petites Sciences" ", Recherches sociographiques, vol. XX, nº 1 (1979), p. 7-39. Nous disons partiellement puisque, selon cette hypothèse, les élites en place auraient, en contrepartie, boudé les sciences. Comme l'a mentionné Raymond Duchesne, cela est loin d'avoir été démontré (voir « D'intérêt public et d'intérêt privé : l'institutionnalisation de l'enseignement et de la recherche scientifiques au Québec (1920-1940) ", dans Yvan Lamonde et Esther Trépanier (dir.), L'avènement de la modernité culturelle au Québec, Québec, IQRC, 1986, p. 195-202).

${ }^{87}$ L'expression est de Descarries-Bélanger, Fournier et Maheu, "Le frère MarieVictorin et "Les Petites Sciences" ".

${ }^{88}$ Faut-il se surprendre que le premier contact qu'eut René Pomerleau avec les champignons ait été tributaire d'un frère des écoles chrétiennes d'origine française et dont la communauté était responsable de son école primaire? Voir Pomerleau, "Le quart de siècle du Cercle des mycologues de Montréal », tapuscrit, p. 3.
} 